\title{
Encochleated Amphotericin B
}

National Cancer Institute

\section{Source}

National Cancer Institute. Encochleated Amphotericin B. NCI Thesaurus. Code C150700.

A proprietary, orally bioavailable, nanoparticle-based encochleated formulation composed of amphotericin B, a polyene antifung al antibiotic produced by Streptomyces nodosus, encochleated within lipid-based crystals, which are composed of multi-layered, rolled-up sheets of soy-derived phosphatidylserine (PS) bilayers and calcium, with broadspectrum antifung al activity. Upon oral administration, the cochleate crystal structure protects amphotericin B from degradation within the gastrointestinal (GI) tract. The lipid structure facilitates uptake into the bloodstream where the nanoparticles are taken up by macrophages and fungal cells though phagocytosis. Amphotericin B is released from the cochleate due to the calcium differential between the inside of the cell, which has low calcium levels, and the outside of the cell, which has high calcium levels. This delivers amphotericin B directly to the fungal-infected site and to its target cells. In turn, amphotericin B binds to ergosterol, an essential component of the fung al cell membrane. This results in depolarization of the cell membrane, alterations in cell membrane permeability, leakage of important intracellular components, and cell rupture. This causes fungal cell death and prevents fungal infections. Compared to amphotericin B alone, which can only be administered intravenously (IV), this formulation permits oral administration and reduces treatment-limiting side effects that are seen with IV amphotericin B, particularly nephro- and hepato-toxicity. 